\title{
Approximating a Convex Body by a Polytope Using the Epsilon-Net Theorem
}

\author{
Márton Naszódi ${ }^{1}$
}

Received: 24 July 2017 / Revised: 13 January 2018 / Accepted: 20 February 2018

C Springer Science+Business Media, LLC, part of Springer Nature 2018

\begin{abstract}
We prove that roughly $\frac{d}{(1-\vartheta)^{d}} \ln \frac{1}{(1-\vartheta)^{d}}$ points chosen uniformly and independently from a centered convex body $K$ in $\mathbb{R}^{d}$ yield a polytope $P$ for which $\vartheta K \subseteq P \subseteq K$ holds with large probability. This gives a joint generalization of results of Brazitikos, Chasapis and Hioni and of Giannopoulos and Milman.
\end{abstract}

Keywords Approximation by polytopes - Convex body · Epsilon-net theorem · Grünbaum's theorem · VC-dimension

Mathematics Subject Classification 52A27 · 52A20

\section{Introduction}

A convex body (i.e., a compact convex set with non-empty interior) in $\mathbb{R}^{d}$ is called centered, if its center of mass is the origin.

We study the following problem. Given a centered convex body $K$ in $\mathbb{R}^{d}$, a positive integer $t \geq d+1$, and $\delta, \vartheta \in(0,1)$. We want to show that under some assumptions on the parameters $d, t, \delta, \vartheta$ (and without assumptions on $K$ ), the convex hull $P$ of $t$ randomly, uniformly and independently chosen points of $K$ contains $\vartheta K$ with probability at least $1-\delta$.

Editor in Charge: János Pach

Márton Naszódi

marton.naszodi@math.elte.hu

1 Department of Geometry, Lorand Eötvös University, Pázmány Péter Sétány 1/C, Budapest 1117 , Hungary 
[4, Thm. 1.1] concerns the case of very rough approximation, that is, where the number $t$ of chosen points is linear in the dimension $d$. It states that the convex hull of $t=\alpha d$ random points in a centered convex body $K$ is a convex polytope $P$ which satisfies $\frac{c_{1}}{d} K \subseteq P$, with probability $1-\delta=1-e^{-c_{2} d}$, where $c_{1}, c_{2}>0$ and $\alpha>1$ are absolute constants. In our first result, we obtain explicit constants.

Theorem 1.1 Let $K$ be a centered convex body in $\mathbb{R}^{d}$. Choose $t=60(d+1)$ points $X_{1}, \ldots, X_{t}$ of $K$ randomly, independently and uniformly. Then

$$
\frac{1}{d} K \subseteq \operatorname{conv}\left\{X_{1}, \ldots, X_{t}\right\} \subseteq K
$$

with probability at least $1-4 e^{-d-1}$.

Another instance of our general problem is [7, Thm. 5.2], which concerns fine approximation, that is, where the number $t$ of chosen points is exponential in the dimension $d$. It states that for any $\delta, \gamma \in(0,1)$, if we choose $t=e^{\gamma d}$ random points in any centered convex body $K$ in $\mathbb{R}^{d}$, then the convex polytope $P$ thus obtained satisfies $c(\delta) \gamma K \subseteq P$, with probability $1-\delta$. We note that it is not included explicitly in the statement of of [7, Thm. 5.2] that it only holds for sufficiently large $d$, that is, when $d>d_{0}$, where $d_{0}$ depends on $\delta$ and $\gamma$. This condition is clearly necessary, as for any $\gamma$ and any $K$, with some positive probability, the origin is not in the convex hull of $t=e^{\gamma d}$ random points in $K$.

[7, Prop. 5.3] follows from the same argument as Theorem 5.2 therein. It states that for any $\delta, \vartheta \in(0,1)$, if we choose $t=c(\delta)\left(\frac{c}{1-\vartheta}\right)^{d}$ random points in any centered convex body $K$ in $\mathbb{R}^{d}$, then the convex polytope $P$ thus obtained satisfies $\vartheta K \subseteq P$, with probability $1-\delta$.

Our main result is the following.

Theorem 1.2 Let $\vartheta \in(0,1), C \geq 2$. Set

$$
t:=\left\lceil C \frac{(d+1) e}{(1-\vartheta)^{d}} \ln \frac{e}{(1-\vartheta)^{d}}\right\rceil .
$$

Then for any centered convex body $K$ in $\mathbb{R}^{d}$, if $t$ points $X_{1}, \ldots, X_{t}$ of $K$ are chosen randomly, independently and uniformly, then

$$
\vartheta K \subseteq \operatorname{conv}\left\{X_{1}, \ldots, X_{t}\right\} \subseteq K
$$

with probability at least $1-\delta$, where

$$
\delta:=4\left[11 C^{2}\left(\frac{(1-\vartheta)^{d}}{e}\right)^{C-2}\right]^{d+1}
$$

By substituting $\vartheta=\frac{1}{d}, C=6$, we obtain Theorem 1.1. 
In order to recover [7, Thm. 5.2], substitute $C=3$ and $\vartheta=c(\delta) \gamma$ in our Theorem 1.2. Then $t \leq e^{3 c(\delta) \gamma d}$, when $d$ is large, and $\delta$ is roughly $e^{-c(\delta) \gamma d^{2}}$. Fixing $c(\delta)=1 / 3$ independently of $\delta$ yields the result.

We recover [7, Prop. 5.3] in a form which is slightly weaker if $\vartheta$ is close to 1 , as follows. In our Theorem 1.2, $t \leq \frac{10 C d^{2}}{(1-\vartheta)^{d+1}}$ (note the exponent $d+1$ instead of $d$ ) and $\delta \leq 11 C^{2} / e^{C-2}$. By setting $C$ sufficiently large (depending on the desired $\delta$ only), we can make the latter as small as required.

We compare our Theorem 1.2 with the main result, [4, Thm. 1.2], which states the following. Let $\beta \in(0,1)$. There exist a constant $\alpha=\alpha(\beta)>1$ depending only on $\beta$ and an absolute constant $c>0$ with the following property. Let $K$ be a centered convex body in $\mathbb{R}^{d}, \alpha d \leq t \leq e^{d}$, and choose $t$ points uniformly distributed in $K$. Then the convex polytope thus obtained contains $\vartheta K$, where $\vartheta=\frac{c \beta \ln (t / d)}{d}$ with probability $1-\delta$, where $\delta \leq \exp \left(-t^{1-\beta} d^{\beta}\right)$.

When $\vartheta$ is of order $1 / d$, the two results are the same up the constants involved, see our Theorem 1.1 and the discussion preceding it. For fine approximation, that is, when $\vartheta$ is a constant, by setting $C=\frac{1}{(1-\vartheta)^{d / 2}}$, we obtain roughly $t \approx \exp (\vartheta d / 2)$ and $\delta \approx \exp \left[-\vartheta d^{2} \exp (\vartheta d / 2)\right]$. In the mean time, [4, Thm. 1.2] gives roughly $t \approx$ $\exp (\vartheta d /(c \beta))$ and $\delta \approx \exp \left[-\exp ((1-\beta) \vartheta d /(c \beta)) d^{\beta}\right]$.

In Sect. 2, we present a generalization of a classical result of Grünbaum [10], according to which any half-space containing the center of mass of a convex body contains at least a $1 / e$ fraction of its volume. In Sect. 3, we state a specific form of the $\varepsilon$-net theorem, a result from combinatorics obtained by Haussler and Welzl [11] building on ideas of Vapnik and Chervonenkis [21], and then refined by Komlós et al. [12]. In Sect. 4, we combine these two to obtain Theorem 1.2. Finally, in Sect. 5, using a recent result of Fradelizi et al. [6], we extend our main result to approximating a linear section of a centered convex body.

For surveys on the topic of approximation of convex bodies by polytopes, cf. [2,5,9], and for some further recent results on approximation in the Banach-Mazur distance (or, geometric distance) when the vertices are not necessarily picked randomly and uniformly from the body, see $[3,16]$.

We note that, in a similar vein, Gordon, Litvak, Pajor and Tomczak-Jaegermann $[8$, Thm. 3.1] showed that if $K$ is an origin-symmetric convex body in $\mathbb{R}^{d}$ and $t=(4 / \varepsilon)^{2 d}$ random points $X_{1}, \ldots, X_{t}$ are chosen from it uniformly and independently, then, with probability larger than $1-\exp \left(-(8 / \varepsilon)^{d} / 2\right)$, these $t$ points form a metric $\varepsilon$-net of $K$ with respect to $K$, that is, $K \subseteq \bigcup_{i=1}^{t}\left(X_{i}+\varepsilon K\right)$. We will use the term ' $\varepsilon$-net' in a different, combinatorial sense, to be defined in Sect. 3.

\section{Convexity: A stability Version of a Theorem of Grünbaum}

Grünbaum's theorem [10] states that for any centered convex body $K$ in $\mathbb{R}^{d}$, and any half-space $F_{0}$ that contains the origin we have

$$
\operatorname{vol}(K) / e \leq \operatorname{vol}\left(K \cap F_{0}\right)
$$


where vol $(\cdot)$ denotes volume.

We say that a half-space $F$ supports $K$ from outside if the boundary of the half-space intersects bd $K$, but $F$ does not intersect the interior of $K$. Lemma 2.1, is a stability version of Grünbaum's theorem.

Lemma 2.1 Let $K$ be a convex body in $\mathbb{R}^{d}$ with centroid at the origin. Let $0<\vartheta<1$, and $F$ be a half-space that supports $\vartheta K$ from outside. Then

$$
\operatorname{vol}(K) \frac{(1-\vartheta)^{d}}{e} \leq \operatorname{vol}(K \cap F) .
$$

Proof Let $F_{0}$ be a translate of $F$ containing $o$ on its boundary, and let $F_{1}$ be a translate of $F$ that supports $K$ from outside. Finally, let $p \in$ bd $F_{1} \cap K$. Then $\vartheta p+(1-\vartheta)\left(K \cap F_{0}\right)$ (that is, the homothetic copy of $K \cap F_{0}$ with homothety center $p$ and ratio $1-\vartheta$ ) is in $K \cap F$. Its volume is $(1-\vartheta)^{d} \operatorname{vol}\left(K \cap F_{0}\right)$, which by $(1)$, is at least $(1-\vartheta)^{d} \operatorname{vol}(K) / e$, finishing the proof.

\section{Combinatorics: The $\varepsilon$-Net Theorem of Haussler and Welzl}

Definition 3.1 Let $\mathcal{F}$ be a family of subsets of some set $U$. The Vapnik-Chervonenkis dimension (VC-dimension, in short) of $\mathcal{F}$ is the maximal cardinality of a subset $V$ of $U$ such that $V$ is shattered by $\mathcal{F}$, that is, $\{F \cap V: F \in \mathcal{F}\}=2^{V}$.

A transversal of the set family $\mathcal{F}$ is a subset $Q$ of $U$ that intersects each member of $\mathcal{F}$.

Let $\varepsilon \in(0,1)$ be given. When $U$ is equipped with a probability measure for which each member of $\mathcal{F}$ is measurable, then a transversal of those members of $\mathcal{F}$ that are of measure at least $\varepsilon$ is called an $\varepsilon$-net.

It follows from Radon's lemma (cf. [13, Thm. 1.3.1], or [19, Thm. 1.1.5]) that if $U$ is any subset of $\mathbb{R}^{d}$, and $\mathcal{F}$ is a family of half-spaces of $\mathbb{R}^{d}$, then the VC-dimension of $\mathcal{F}$ is at most $d+1$.

The $\varepsilon$-Net Theorem was first proved by Haussler and Welzl [11], and then improved by Komlós et al. [12]. We state a slightly weaker form of Theorem 3.1 of [12] than the original, in order to have an explicit bound on the probability $\delta$ of failure.

Lemma 3.2 ( $\varepsilon$-Net Theorem). Let $0<\varepsilon<1 / e, C \geq 2$, and let $D$ be a positive integer. Let $\mathcal{F}$ be a family of some measurable subsets of a probability space $(U, \mu)$, where the probability of each member $F$ of $\mathcal{F}$ is $\mu(F) \geq \varepsilon$. Assume that the $V C$ dimension of $\mathcal{F}$ is at most D. Set

$$
t:=\left\lceil C \frac{D}{\varepsilon} \ln \frac{1}{\varepsilon}\right\rceil .
$$

Choose $t$ elements $X_{1}, \ldots, X_{t}$ of $V$ randomly, independently according to $\mu$. Then $\left\{X_{1}, \ldots, X_{t}\right\}$ is a transversal of $\mathcal{F}$ with probability at least $1-\delta$, where

$$
\delta:=4\left[11 C^{2} \varepsilon^{C-2}\right]^{D}
$$


Proof We provide an outline of the first, conceptual part of the proof closely following [17, Thm. 15.5]. Then, we continue with a detailed computation to obtain the bound on the probability stated in Lemma 3.2.

Let $T>t$ be an integer, to be set later. We select (with repetition) independently $t$ random elements of $U$ with respect to $\mu$, call it the first sample, and denote it by $x$. Then, we choose another $T-t$ elements, call it the second sample, and denote it by $y$. For any $F \in \mathcal{F}$, and any finite sequence $w$ of elements of $U$, let $I(F, w)$ denote the number of elements of $w$ in $F$ with multiplicity. Let $m_{F}$ denote the median of $I(F, y)$.

Note that $I(F, y)$ is a binomial variable, and hence, its mean and median are close to each other. More precisely,

$$
m_{F} \geq(T-t) \varepsilon-1 .
$$

It is not hard to see that

$$
\mu(\exists F \in \mathcal{F}: I(F, x)=0) \leq 2 \mu\left(\exists F \in \mathcal{F}: I(F, x)=0 \text { and } I(F, y) \geq m_{F}\right) .
$$

Denote the concatenation of the two sequences $x$ and $y$ by $\overline{x y}$. Fix any length $T$ sequence $z$ of elements of $U$.

It is simple to obtain a bound on the following conditional probability:

$$
\begin{aligned}
\mu(\exists F \in \mathcal{F}: I(F, x)= & \text { Oand } \left.I(F, y) \geq m_{F} \mid \overline{x y}=z\right) \\
& \leq \chi\left[I(F, z) \geq m_{F}\right]\left(1-\frac{t}{T}\right)^{m_{F}}
\end{aligned}
$$

where $\chi$ denotes the indicator function of an event, that is, it is one if the event holds, and zero otherwise.

The key idea follows. Consider $z$ as a set. Then, by the Shatter function lemma (cf. of [17, Thm. 15.4] or [13, Lem. 10.2.5]) proved independently by Shelah [20], Sauer [18] and Vapnik and Chervonenkis [21], $z$ has at most

$$
\sum_{i=0}^{D}\left(\begin{array}{l}
T \\
i
\end{array}\right)
$$

distinct intersections with members of $\mathcal{F}$. Thus by (3) and (5), we have

$$
\mu\left(\exists F \in \mathcal{F}: I(F, x)=0 \text { and } I(F, y) \geq m_{F} \mid \overline{x y}=z\right) \leq \sum_{i=0}^{D}\left(\begin{array}{l}
T \\
i
\end{array}\right)\left(1-\frac{t}{T}\right)^{(T-t) \varepsilon-1}
$$

Let $E$ be the 'bad' event, that is, when $\left\{X_{1}, \ldots, X_{t}\right\}$ is not a transversal of $\mathcal{F}$. So far, by (4) and (6), we obtained that for any integer $T>t$, we have that the probability of the event $E$ is

$$
\mu(E)<2 \sum_{i=0}^{D}\left(\begin{array}{l}
T \\
i
\end{array}\right)\left(1-\frac{t}{T}\right)^{(T-t) \varepsilon-1}
$$


From this point on, we describe the computations in detail, in order to obtain the bound on the probability stated in Lemma 3.2.

We set $T=\left\lfloor\frac{\varepsilon t^{2}}{D}\right\rfloor$ and use $\sum_{i=0}^{D}\left(\begin{array}{c}T \\ i\end{array}\right) \leq\left(\frac{e T}{D}\right)^{D}$, to obtain that

$$
\begin{aligned}
\mu(E) & <2\left(\frac{e T}{D}\right)^{D}\left(1-\frac{t}{T}\right)^{(T-t) \varepsilon-1}<2\left(\frac{e \varepsilon t^{2}}{D^{2}}\right)^{D}\left(1-\frac{D}{\varepsilon t}\right)^{\left(\varepsilon t^{2} / D-t-1\right) \varepsilon-1} \\
& <2\left(\frac{e \varepsilon t^{2}}{D^{2}}\right)^{D} e^{-\varepsilon t+D+D / t+D /(\varepsilon t)}
\end{aligned}
$$

which, after substituting the expression for $t$ in some places and using $\varepsilon<1 / e$, is at most

$$
\left(2 e^{1 / C+1 /(e C)}\right)\left(\frac{e^{2} \varepsilon t^{2}}{D^{2}}\right)^{D} \varepsilon^{C D}
$$

which, using $C \geq 2$ is at most

$$
\left(2 e^{1 / C+1 /(e C)}\right)\left(\frac{e^{2}(1+1 /(2 e))^{2} C^{2} \ln ^{2}(1 / \varepsilon)}{\varepsilon}\right)^{D} \varepsilon^{C D}<4\left(11 C^{2} \varepsilon^{C-2}\right)^{D}
$$

completing the proof of Lemma 3.2.

For more on the theory of $\varepsilon$-nets, see $[1,13,15,17]$.

\section{Proof of Theorem 1.2}

Proof of Theorem 1.2 We consider the following set system on the base set $K$ :

$$
\mathcal{F}:=\{K \cap F: F \text { is a half-space that supports } \vartheta K \text { from outside }\}
$$

Clearly, the VC-dimension of $\mathcal{F}$ is at most $D:=d+1$. Let $\mu$ be the Lebesgue measure restricted to $K$, and assume that $\operatorname{vol}(K)=1$, that is, that $\mu$ is a probability measure. By (2), we have that each set in $\mathcal{F}$ is of measure at least $\varepsilon:=\frac{(1-\vartheta)^{d}}{e}$. Lemma 3.2 yields that if we choose $t$ points of $K$ independently with respect to $\mu$ (that is, uniformly), then with probability at least $1-\delta$, we obtain a set $Q \subseteq K$ that intersects every member of $\mathcal{F}$. The latter is equivalent to $\vartheta K \subseteq$ conv $Q$, completing the proof.

\section{Approximating a Section of a Convex Body}

Let $K$ be a centered convex body in $\mathbb{R}^{d}$, and $V$ a linear subspace of $\mathbb{R}^{d}$. Now, $K \cap V$ may not be centered however, we may still want to approximate $K \cap V$ with a polytope $P \subset K \cap V$ such that $\vartheta(K \cap V) \subset P$ for some not too small $\vartheta$.

The main result of [6] (for further results, see also [14]) states that there is an absolute constant $c>0$ such that for every centered convex body $K$ in $\mathbb{R}^{d}$, every 
$(d-k)$-dimensional linear subspace $V$ of $\mathbb{R}^{d}, 0 \leq k \leq d-1$, and any $u \in V$ unit vector, we have

$$
\operatorname{vol}_{d-k}\left(K \cap V \cap u^{+}\right) \geq \frac{c}{(k+1)^{2}}\left(1+\frac{k+1}{d-k}\right)^{-(d-k-2)} \operatorname{vol}_{d-k}(K \cap V),
$$

where $u^{+}=\left\{x \in \mathbb{R}^{d}:\langle u, x\rangle \geq 0\right\}$ is the half-space with inner normal vector $u$.

Using this result, our proof of Theorem 1.2 immediately yields the following.

Theorem 5.1 Let $\vartheta \in(0,1), C \geq 2$. Let $K$ be a centered convex body in $\mathbb{R}^{d}$ and $V$ be $(d-k)$-dimensional linear subspace of $\mathbb{R}^{d}$ with $0 \leq k \leq d-1$. Set

$$
t:=\left\lceil C \frac{(d-k+1)(k+1)^{2}}{c\left(1+\frac{k+1}{d-k}\right)^{d-k-2}(1-\vartheta)^{d-k}} \ln \frac{(k+1)^{2}}{c\left(1+\frac{k+1}{d-k}\right)^{d-k-2}(1-\vartheta)^{d-k}}\right\rceil,
$$

where $c$ is the universal constant from (7). Choose t points $X_{1}, \ldots, X_{t}$ of $K \cap V$ randomly, independently and uniformly with respect to the $(d-k)$-dimensional Lebesgue measure on $V$. Then

$$
\vartheta(K \cap V) \subseteq \operatorname{conv}\left\{X_{1}, \ldots, X_{t}\right\} \subseteq K \cap V
$$

with probability at least $1-\delta$, where

$$
\delta:=4\left[11 C^{2}\left(\frac{c\left(1+\frac{k+1}{d-k}\right)^{d-k-2}(1-\vartheta)^{d-k}}{(k+1)^{2}}\right)^{C-2}\right]^{d-k+1} .
$$

Acknowledgements The author thanks Nabil Mustafa for enlightening conversations on the $\varepsilon$-net theorem and topics around it. I also thank the anonymous referees whose remarks helped fix some errors and improve the presentation. The research was partially supported by the National Research, Development and Innovation Fund Grant K1 19670, and by the János Bolyai Research Scholarship of the Hungarian Academy of Sciences, and by the ÚNKP-17-4 New National Excellence Program of the Ministry of Human Capacities. Part of the work was carried out during a stay at EPFL, Lausanne at János Pach's Chair of Discrete and Computational Geometry supported by the Swiss National Science Foundation Grants 200020-162884 and 200021-165977.

\section{References}

1. Alon, N., Spencer, J.H.: The Probabilistic Method, 4th edn. Discrete Mathematics and Optimization. Wiley, Hoboken (2016)

2. Bárány, I.: Random polytopes, convex bodies, and approximation. In: Weil, W. (ed.) Stochastic Geometry. Lecture Notes in Mathematics, vol. 1892, pp. 77-118. Springer, Berlin (2007)

3. Barvinok, A.: Thrifty approximations of convex bodies by polytopes. Int. Math. Res. Not. IMRN 2014(16), 4341-4356 (2014)

4. Brazitikos, S., Chasapis, G., Hioni, L.: Random approximation and the vertex index of convex bodies. Arch. Math. (Basel) 108(2), 209-221 (2017). https://doi.org/10.1007/s00013-016-0975-2

5. Bronshter̆ n, E.M.: Approximation of convex sets by polyhedra. J. Math. Sci. (N.Y.) 153(6), 727-762 (2008)

6. Fradelizi, M., Meyer, M., Yaskin, V.: On the volume of sections of a convex body by cones. Proc. Amer. Math. Soc. 145(7), 3153-3164 (2017) 
7. Giannopoulos, A.A., Milman, V.D.: Concentration property on probability spaces. Adv. Math. 156(1), 77-106 (2000)

8. Gordon, Y., Litvak, A.E., Pajor, A., Tomczak-Jaegermann, N.: Random $\varepsilon$-nets and embeddings in $l_{\infty}^{N}$. Stud. Math. 178(1), 91-98 (2007)

9. Gruber, P.M.: Aspects of approximation of convex bodies. In: Gruber, P.M., Wills, J.M. (eds.) Handbook of Convex Geometry, vol. A,B, pp. 319-345. Elsevier, Amsterdam (1993)

10. Grünbaum, B.: Partitions of mass-distributions and of convex bodies by hyperplanes. Pac. J. Math. 10, 1257-1261 (1960)

11. Haussler, D., Welzl, E.: $\varepsilon$-nets and simplex range queries. Discrete Comput. Geom. 2(2), 127-151 (1987)

12. Komlós, J., Pach, J., Woeginger, G.: Almost tight bounds for $\varepsilon$-nets. Discrete Comput. Geom. 7(2), 163-173 (1992)

13. Matoušek, J.: Lectures on Discrete Geometry. Graduate Texts in Mathematics, vol. 212. Springer, New York (2002)

14. Meyer, M., Nazarov, F., Ryabogin, D., Yaskin, V.: Generalized Grünbaum inequality. http://arxiv.org/ abs/1706.02373 [math] (2017)

15. Mustafa, N.H., Varadarajan, K.: Epsilon-approximations \& epsilon-nets. In: Jacob, J.O., Goodman, E., Tóth, C.D. (eds.) Handbook of Discrete and Computational Geometry. CRC Press LLC, Boca Raton (2018)

16. Naszódi, M., Nazarov, F., Ryabogin, D.: Fine approximation of convex bodies by polytopes. http:// arxiv.org/abs/1705.01867 [math] (2017)

17. Pach, J., Agarwal, P.K.: Combinatorial Geometry. Wiley, New York (1995)

18. Sauer, N.: On the density of families of sets. J. Comb. Theory Ser. A 13, 145-147 (1972)

19. Schneider, R.: Convex Bodies: The Brunn-Minkowski Theory. In: Encyclopedia of Mathematics and its Applications, vol. 151, Cambridge University Press, Cambridge (2014)

20. Shelah, S.: A combinatorial problem; stability and order for models and theories in infinitary languages. Pac. J. Math. 41, 247-261 (1972)

21. Vapnik, V.N., Červonenkis, A.J.: On the Uniform Convergence of Relative Frequencies of Events to Their Probabilities, vol. 181(4), pp. 781-783. Doklady Akademii Nauk (1968) (in Russian) 